\title{
RELAÇÃO ENTRE O CONSUMO ALMENTAR E ATIVIDADE FÍSICA COM O ÍNDICE DE MASSA CORPORAL EM FUNCIONÁRIOS UNIVERSITÁRIOS ${ }^{1}$
}

\section{RBATIONSHIP BETWMN FOOD INTAKE AND PHYSICAL ACTMMTY WTH BODY MASS INDEX IN UNIVERSITY EMPLOYERS}

\author{
Rosely SICHIERI ${ }^{2}$ \\ Rosângela Alves PEREIRA ${ }^{3}$ \\ Vânia Maria Ramos de MARINS ${ }^{4}$ \\ Rita de Cássia PERREШ ${ }^{3}$ \\ Maria Auxiliadora Santa Cruz COELHO 4 \\ Maria DelCarmen MOLNA ${ }^{3}$
}

\begin{abstract}
RESUMO
Nasúltimas duas décadas observou-se um importante aumento da prevalência de obesidade no Brasil, contudo não existem dados populacionais atualizados sobre ingestão de alimentos e atividades físicas, que seriam em última instância os fatores que poderiam explicar este aumento de obesidade. Estudou-se a associação entre o consumo de calorias e gordura e a prática de atividades físicas com o índice de massa corporal, em um estudo transversal com 91 funcionários universitários. O consumo alimentar foi avaliado por um questionário semi-quantitativo de freqüência de consumo de alimentos e por quatro recordatórios de 24 horas. Peso e altura foram medidos e calculou-se o índice de massa corporal por meio da relação peso/altura em $\mathrm{kg} / \mathrm{m}^{2}$. As atividades físicas desenvolvidas no trabalho, no lazer e no deslocamento para o trabalho e/ou escola no mês que antecedeu a pesquisa foram investigadas através de questionário. Estimou-se o gasto energético associado às atividades físicas, através de equações propostas pela Organização Mundial da Saúde. Em análise linear multivariada associaram-se positivamente ao índice de massa corporal a idade $(p=0,02)$, pertencer ao grupo de funcionários de nível médio e de apoio, comparados aos professores $(p=0,004)$ e ser do sexo feminino $(p=0,04)$. Ainda, nesta análise, um aumento de 500 calorias no gasto energético estimado equivaleu a uma redução de aproximadamente $2 \mathrm{~kg}$ no peso de um indivíduo com $1,60 \mathrm{~m}(p=0,008)$. Para os dois métodos usados para avaliação de consumo, a ingestão de calorias e de gordura associaram-se, significativamente, com o índice de massa corporal $(p<0,05)$, porém esta associação foi modificada pelo sexo, sendo negativa para as mulheres e fracamente positiva para os homens. Este estudo mostrou que o sexo feminino, nível socioeconômico
\end{abstract}

\footnotetext{
( (1) Trabalho financiado pelo CNPq e pelo Instituto Nacional de Alimentação e Nutrição, Ministério da Saúde; Apresentado no American Society of Nutrition Meeting, San Diego, 1995.

(2) Médica, Professora do Instituto de Medicina Social, Universidade Estadual do Rio de Janeiro, a quem toda correspondência deve ser endereçada, Rua São Francisco Xavier, 524, $7^{\circ}$ andar, 20551-030, Rio de Janeiro, RJ.

(3) Nutricionistas, Professoras do Instituto de Nutrição, Universidade Federal do Rio de Janeiro.

(4) Nutricionistas, Professoras da Faculdade de Nutrição, Universidade Federal Fluminense, Niterói, RJ.
} 
e o gasto energético são preditivos de obesidade e sugere que mulheres obesas subestimam seu consumo alimentar.

Termos de indexação: obesidade, índice de massa corporal, consumo de alimentos, estudos transversais, hábitos alimentares, atividade física.

\begin{abstract}
Obesity has been increasing in Brazil in the last two decades, but there are no recent population data on dietary intake and physical activity, the two major components of body weight gain. The association of food intake (calorie and fat) and energy expenditure with body mass index (BMI) was studied in 91 university employers. Food intake was estimated by the means of four 24-hour recalls and by a semi-quantitative food frequency questionnaire developed using the most frequent consumed Brazilian foods. Weight and height were measured and the body mass index was calculated by dividing the weight in $\mathrm{kg}$ by the height in $\mathrm{m}^{2}$. Physical activities developed in the previous month were estimated through a questionnaire that included activities developed at work, in the spare time and when commuting to work or school. Energy expenditure estimation was based on the WHO equations. Multivariate linear analysis having BMI as the dependent variable showed positive associations with age ( $p=0.02)$, occupation (support staff being heavier than professors, $p=0.004$ ), sex (females were heavier than males, $p=0.04$ ). Furthermore, the daily increase of 500 calories in the energy expenditure was associated to a weight reduction of approximately $2 \mathrm{~kg}$ in an individual with a height of $1.60 \mathrm{~m}(p=0.008)$. For both methods used to estimate the food intake (24-hour recall and food frequency questionnaire), energy intake and fat intake were associated to BMI $(p<0.05)$, but sex modified these associations. For women there was a negative association between fat and energy intake and BMI, whereas for men this association was positive but irrelevant. This study indicated an increased risk of obesity with age, and an increased risk for staff employers and females. A possible misclassification of food intake among obese female was also suggested.
\end{abstract}

Index terms: obesity, body mass index, food consumption, cross-sectional studies, food habits, physical activity.

\section{INTRO DUÇÃO}

OBrasil vem apresentando nas últimas décadas importante alteração do padrãonutricional. A maioria das pesquisas produzidas nos últimos dez anos no Brasil apontaram para o crescimento da prevalência de obesidade em todas as regiões do país, nas diferentes classes sociais e atingindo de forma importante a população mais pobre (ANJOS et al, 1992; MONDINI \& MONTEIRO, 1994; SICHIERI et al., 1994, MONTEIRO et al., 1995). Justifica-se, portanto, tentar buscar os fatores associados a obesidade em nosso meio, que permitiriam estabelecer estratégias de intervenção para o enfrentamento deste importante problema de saúde pública.

Sabe-se que na gênese da obesidade dois fatores desempenham papel primordial; de um lado o consumo alimentar e de outro o gasto energético. Embora estes fatores não sejam de fácil mensuração, vários estudos de âmbito populacional têm buscado formas adequadas de medi-los. Em relação ao consumo alimentar os questionários de freqüência de consumo (QFCA) têm sido freqüentemente utilizados por serem de baixo custo e permitirem a aplicação em amplos estudos populacionais (WILLETT, 1990; WILLETT, 1994). Os QFCA são, particularmente úteis, quando o objeto da coleta de dados é de estabelecer associações entre o consumo e alguma solução, pois o que se espera é que os instrumentos de avaliação da ingestão individual de alimentos sejam capazes de classificar os indivíduos num determinado gradiente de consumo de alimentos, nutrientes ou energia (NATIONAL..., 1989; MARTIN-MORENO et al., 1993). 
A associação entre o consumo alimentar e medidas de sobrepeso ou obesidade não é de fácil mensuração. Associações negativas têm sido observadas entre consumo de energia e obesidade (BRAITMAN et al., 1985; ROMIEU et al., 1988), bem como entre o consumo de energia e doenças cardiovasculares (FEHILY et al., 1993). Uma possível explicação para esta não associação é que a atividade física é uma variável de confusão importante (GORDON et al., 1981; NATIONAL..., 1989b; FEHILY et al., 1993, SALTZMAN \& ROBERTS, 1995).

A associação negativa entre consumo alimentar e obesidade poderia ser também explicada pela existência de um viés importante de informação, principalmente entre as mulheres com sobrepeso e ou obesidade (PRENTICE et al., 1986; SCHOELLER, 1990; LICHTMAN et al., 1992; HEITMANN \& LISSNER, 1996). Nesta perspectiva somente estudos longitudinais, que avaliassem o consumo alimentar antes do aparecimento da obesidade, conseguiriam avaliar a associação entre consumo e medidas de sobrepeso ou obesidade.

Este estudo avaliou o consumo alimentar em funcionários universitários e sua associação com o índice de massa corporal (IMC), controlando para a estimativa de gasto energético que, teoricamente, tanto é preditiva dos valores de IMC, quanto importante variável de confusão da associação entre consumo de energia e obesidade.

\section{MATERIAL E MÉTODO}

O consumo alimentar foi obtido através de dois métodos: um questionário de freqüência do consumo de alimentos (QFCA) e inquéritos recordatórios de 24 horas. O modelo da análise incorporou também as variáveis idade e o nível socioeconômico, uma vez que estes fatores têm sido associados com o aumento do IMC (COITINHO et al., 1991; SICHIERI et al., 1992; SICHIERI et al., 1994). O consumo alimentar foi avaliado em relação a ingestão de gorduras e calorias.

Foram selecionados para o estudo 120 funcionários universitários, sendo 60 trabalhadores em atividades de apoio e 60 servidores de nível superior. Os trabalhadores em atividades de apoio foram selecionados da lista de 45 funcionários da manutenção e as mulheres, entre as do setor de limpeza, que totalizavam 77 mulheres. Para os professores o total de homens era de 386 e o de mulheres 434. Amostragem sistemática foi realizada a partir de listagem de servidores cedida pela administração. Dos 120 funcionários inicialmente sorteados foram investigados 91 trabalhadores (46 homens e 45 mulheres), com idades entre 20 e 65 anos, incluindo professores e servidores técnicoadministrativos de nível superior $(n=45)$ e trabalhadores de apoio $(n=46)$. Estimou-se inicialmente que as perdas seriam de $20 \%$.

Foram coletados dados sobre o consumo de alimentos através do QFCA e de quatro inquéritos recordatórios de 24 horas, e foram solicitadas informações a respeito da intensidade da atividade física no trabalho, no lazer e nos deslocamentos para o trabalho ou escola, a fim de proceder-se a uma estimativa do gasto energético. Foram, ainda, obtidas informações sobre sexo, idade, renda, ocupação e escolaridade.

A coleta de dados foi realizada entre os meses de setembro e outubro de 1994 pelas pesquisadoras e bolsistas envolvidas na pesquisa, todas com formação específica na área de Nutrição. Os participantes compareceram a duas entrevistas, numa das quais era realizado o exame antropométrico.

As informações sobre o consumo de alimentos obtidas através do QFCA, semi-quantitativo incluíam as porções freqüentemente utilizadas segundo PINHEIRO et al. (1993) e a freqüência de consumo por dia, semana e mês. A lista de alimentos era composta por 64 itens e as quantidades diárias consumidas foram obtidas multiplicando-se as porções pela freqüência de consumo. Após a aplicação do QFCA eram realizadas as medidas antropométricas e aplicado o questionário geral e de atividades físicas, epor último eram realizados os inquéritos alimentares.

Os quatro inquéritos recordatórios referiam-se ao consumo nas 24 horas anteriores à primeira entrevista. Os inquéritos incluíam sempre um sábado e/ou um domingo. Após 2 a 4 dias, os participantes eram novamente entrevistados para outros 2 recordatórios de 24 horas relativos a diferentes dias da semana. Estes recordatórios foram utilizados para a validação do QFCA, cujos resultados serão descritos a seguir (SICHIERI et al., 1995a, b).

Para o cômputo de energia e nutrientes dos recordatórios de 24 horas, utilizou-se um programa 
de entrada de dados desenvolvido pelo Instituto de Nutrição da Universidade Federal do Rio de Janeiro, o SISNUT (PYRRO \& LACERDA, 1994). Para o QFCA desenvolveu-se um programa em SAS (Statistical Analysis System-SAS, version.6.4), utilizando o mesmo banco de dados de composição do SISNUT (STATITICAL..., 1995).

Foram tomadas as medidas da massa corporal, estatura, e prega cutânea triciptal. A prega cutânea tirada com o medidor da marca Lange. Para caracterizar o estado nutricional, calculou-se o IMC (massa corporal (em kg) dividida pela estatura $\left(\mathrm{em} \mathrm{m}^{2}\right)$ ). Utilizou-se o ponto de corte de $27 \mathrm{~kg} / \mathrm{m}^{2}$ para definir excesso de peso, que correlaciona-se de forma importante com o aumento da mortalidade(SICHIERI et al., 1992).

A intensidade da atividade física no trabalho foi classificada em muito leve, leve e pesada, conforme proposta da Organização Mundial da Saúde (WORLD..., 1985) e a partir desta classificação estimou-se o requerimento energético para oito horas de trabalho. O requerimento energético diário total foi estimado através das equações de predição do metabolismo basal (WORLD..., 1985), que consideram idade e sexo, conforme publicação da National Academy of Science (NATIONAL..., 1989a). Uma variável totalizadora da atividade física foi estimada a partir dos múltiplos da taxa metabólica basal para o dispêndio de energia segundo as horas utilizadas na realização de diferentes atividades referidas na entrevista.

A estratificação dos indivíduos examinados considerou o gênero e dois grupos ocupacionais: grupo de nível de apoio e grupo de nível superior. A variável grupo ocupacional foi considerada, portanto, indicadora do nível socioeconômico uma vez que encontra-se intensamente relacionada com a renda, a escolaridade e a ocupação, que são três variáveis freqüentemente utilizadas na definição de nível socioeconômico (WINKLEBY et al., 1992).

Foram calculadas médias e desvios-padrão das variáveis contínuas e freqüências absolutas e relativas das variáveis discretas, considerando os estratos de sexo e grupo ocupacional. As médias das variáveis antropométricas, renda e idade foram testadas através de teste $\boldsymbol{t}$ de Student. As medidas da atividade física foram comparadas através de análise de variância ou teste do qui-quadrado.
A associação entre gasto energético e o IMC foi avaliada através de análise multivariada. Considerou-se como variável dependente o IMC e a estimativa do gasto energético uma das variáveis independentes. $\mathrm{O}$ valor da estimativa do gasto energético foi dividido pelo peso individual, pois sendo o IMC um indicador de peso relativo, associá-lo a uma variável também diretamente relacionada ao peso, era, a priori, definir uma associação positiva entre o IMC e o gasto energético.

Foi avaliada, também, a associação do consumo alimentar com o IMC, ajustada pela estimativa de gasto energético que, teoricamente, tanto é variável preditora dos valores de IMC, quanto importante variável de confusão da associação entre consumo de energia e obesidade. O modelo da análise incorporou também as variáveis idade e ocupação.

O IMC por apresentar distribuição bastante desviada para a direita foi transformado no seu logaritmo neperiano. Os modelos com valores transformados e sem transformação foram semelhantes, sendo apresentados somente os valores não transformados pela sua maior facilidade de interpretação.

As variáveis consumo de calorias e consumo de gordura, dado sua colinearidade, foram analisadas em modelos separados. Foram testadas como variáveis modificadoras de efeito o grupo ocupacional (nível médio e de apoio e superior) e o gênero, pela introdução nos modelos de variáveis que resultavam da multiplicação do grupo ocupacional e gênero entre si e, destas duas variáveis, com o consumo de gordura e o consumo de energia. Foram mantidos nos modelos somente os termos de interação que se associaram significantemente $(\mathrm{p} \leq 0.05)$ com o IMC. As variáveis consideradas de confusão foram mantidas no modelo independentemente de sua significância estatística.

\section{RESULTADOS}

Responderam completamente os questionários 91 indivíduos. As perdas foram ligeiramente maior do que o esperado e totalizaram $25 \%$, incluindo cinco indivíduos que não retornaram para a segunda entrevista.

A idade média dos homens de nível superior foi maior que a idade média dos de nível de apoio. A 
média da renda dos trabalhadores de nível superior foi significativamente mais elevada que a renda dos de nível médio e de apoio, tanto entre os homens quanto entre as mulheres $(p<0,01)$ (Tabela 1$)$. A Tabela 1 demonstra, ainda, que a renda média dos servidores de nível superior não apresentou diferenças marcantes entre homens e mulheres. Entretanto, entre os trabalhadores de nível de apoio o diferencial de renda entre os gêneros foi importante. A estatura média dos homens foi maior que a das mulheres e a estatura média dos trabalhadores de nível superior foi $5 \mathrm{~cm}$ maior que a dos trabalhadores de nível de apoio, nos dois gêneros. Para o IMC somente entre as mulheres observou-se diferença entre os grupos de ocupação. Embora não apresentando diferença em relação aos valores médios de IMC, os homens com ocupação de apoio apresentaram prega tricipital significativamentemaior.

Tabela 1. Médias e desvios-padrão para idade, renda, indicadores antropométricos e de atividade física, segundo o sexo e a ocupação. Rio de Janeiro - 1994.

\begin{tabular}{|c|c|c|c|c|}
\hline \multirow[b]{2}{*}{ Características investigadas } & \multicolumn{2}{|c|}{ Homens } & \multicolumn{2}{|c|}{ Mulheres } \\
\hline & $\begin{array}{l}\text { Nível superior } \\
\qquad(n=23)\end{array}$ & $\begin{array}{l}\text { Nível médio e } \\
\text { apoio }(n=22)\end{array}$ & $\begin{array}{l}\text { Nível superior } \\
\qquad(n=22)\end{array}$ & $\begin{array}{l}\text { Nível médio e } \\
\text { apoio }(n=24)\end{array}$ \\
\hline Idade (anos) & $44,8 \pm 9,2$ & $42,1 \pm 14,1^{* *}$ & $39,4 \pm 7,5$ & $37,7 \pm 16,0$ \\
\hline Renda (em reais) ${ }^{1}$ & $784,00 \pm 673,00$ & $178,00 \pm 142,00^{*}$ & $708,00 \pm 477,00$ & $70,60 \pm 50,10^{*}$ \\
\hline $\operatorname{IMC}\left(\mathrm{kg} / \mathrm{m}^{2}\right)^{2}$ & $25,0 \pm 3,5$ & $25,2 \pm 4,3$ & $24,0 \pm 3,7$ & $26,3 \pm 4,4 * * *$ \\
\hline Prega Triciptal (em mm) & $11,3 \pm 4,8$ & $15,4 \pm 7,9 * *$ & $19,6 \pm 6,2$ & $18,9 \pm 5,9$ \\
\hline Estatura (em cm) & $172,3 \pm 6,6$ & $167,4 \pm 7,3^{* *}$ & $159,0 \pm 6,2$ & $154,0 \pm 5,6^{*}$ \\
\hline Tempo de locomoção à pé (minutos/dia) & $11 \pm 14$ & $28 \pm 20 *$ & $8 \pm 11$ & $31 \pm 19 *$ \\
\hline \multicolumn{5}{|c|}{ Intensidade da atividade física no trabalho - n (\%) } \\
\hline Muito Leve & $16(69,6)$ & $15(68,2)$ & $17(77,3)$ & $1(4,2)$ \\
\hline Leve & $7(30,4)$ & $5(22,7)$ & $5(22,7)$ & $22(91,6)$ \\
\hline Pesada & 0 & $2(9,1)$ & 0 & $1(4,2)$ \\
\hline \multicolumn{5}{|c|}{ Prática de atividade física associada ao lazer - n (\%) } \\
\hline Não & $8 \pm 34,7$ & $10 \pm 45,5$ & $16(72,7)$ & $24(100,0)$ \\
\hline Sim & $15 \pm 65,2$ & $12 \pm 54,5$ & $6(27,3)$ & \\
\hline
\end{tabular}

(1) Renda individual no mês anterior, na época R \$1,00 (um real) valia U\$ 1,00 (um dólar americano) e o salário mínimo era de $\mathrm{R} \$ 100,00$ (cem reais).

(2) Índice de Massa Corporal = massa $(\mathrm{Kg}) /$ estatura $\left(\mathrm{m}^{2}\right)$.

(*) $\mathrm{p} \leq 0,01 ; * * \mathrm{p} \leq 0,05 ; * * * 0,05<\mathrm{p}<0,10$, associado ao teste $\mathrm{t}$, segundo ocupação.

Os trabalhadores de apoio gastavammais tempo locomovendo-se a pé para o trabalho do que os de nível superior, em ambos os gêneros e nenhum dos profissionais de nível superior exercia atividade física associada ao trabalho considerada pesada. Mesmo entre os trabalhadores de nível médio e de apoio, somente dois homens e uma mulher tiveram atividade laboral classificada como pesada conforme os critérios adotados pela Organização Mundial de Saúde (WORLD..., 1985). Nenhuma mulher do grupo de nível médio e apoio referiram a prática de atividade física no lazer (Tabela 1).

A prevalência do excesso de peso (IMC > $27 \mathrm{~kg} / \mathrm{m}^{2}$ ) no grupo estudado foi de $30 \%$ entre os homens e de $33 \%$ entre as mulheres, sendo que entre os trabalhadores de nível médio e de apoio o excesso 
de peso apareceu com freqüência quase 3 vezes maior (45\% entre homens e mulheres) do que entre os profissionais de nível superior. Dentre estes últimos, $15 \%(n=3)$ dos homens e $18 \%(n=4)$ das mulheres apresentavam excesso de peso.

Os dados dos quatro inquéritos recordatórios foram trabalhados em conjunto, permitindo estimar as médias de consumo de energia e gordura, constante da Tabela 2. Para a avaliação de consumo por meio do método recordatório as médias de consumo de energia foram maiores entre os trabalhadores de nível superior para ambos os sexos e o consumo de gordura só foi estatisticamente maior para as mulheres. As médias avaliadas através do QFCA segundo ocupação, não foram significativamente diferentes.

O gasto energético diário estimado foi significativamente maior entre as mulheres de nível médio e de apoio (2 281kcal) comparadas às de nível superior (1870kcal) e para os homens esteve próximo de $2500 \mathrm{kcal}$, nos dois grupos (Tabela 2). O percentual médio de calorias fornecidas pelas gorduras foi semelhante entre os homens (31,0\%), porém entre as mulheres, os dados demonstraram que as profissionais de nível superior consumiam proporcionalmente maior quantidade de gorduras $(35,0 \%)$ que as de nível médio e de apoio 28,0\%).

Tabela 2. Médias e desvios-padrão do consumo de energia e gordura estimados pela média dos quatro inquéritos recordatórios e pelo questionário de freqüência do consumo de alimentos (QFCA) e estimativa do gasto energético segundo o sexo e a ocupação. Rio de Janeiro - 1994.

\begin{tabular}{|c|c|c|c|c|}
\hline & \multicolumn{2}{|c|}{ Homens } & \multicolumn{2}{|c|}{ Mulheres } \\
\hline & $\begin{array}{l}\text { Nível superior } \\
\qquad(n=23)\end{array}$ & $\begin{array}{l}\text { Nível médio e } \\
\text { apoio }(n=22)\end{array}$ & $\begin{array}{l}\text { Nível superior } \\
\qquad(\mathrm{n}=22)\end{array}$ & $\begin{array}{l}\text { Nível médio e } \\
\text { apoio }(n=24)\end{array}$ \\
\hline & \multicolumn{4}{|c|}{ Média dos 4 recordatórios } \\
\hline energia (kcal) & $2530 \pm(265)$ & $2005 \pm 899 * *$ & $1693 \pm 328$ & $1436 \pm 450^{* *}$ \\
\hline \multirow[t]{2}{*}{ gorduras (g) } & $87,9 \pm(26,0)$ & $69,2 \pm 45,0$ & $65,3 \pm 16,0$ & $44,2 \pm 18,9 *$ \\
\hline & \multicolumn{4}{|c|}{ QFCA } \\
\hline energia (kcal) & $2122 \pm 1119$ & $2214 \pm 784$ & $1560 \pm 663$ & $1763 \pm 645$ \\
\hline gorduras (g) & $57,7 \pm 32,7$ & $66,6 \pm 48,7$ & $37,8 \pm 21,6$ & $38,2 \pm . .19,5$ \\
\hline Gasto energético estimado & $2479 \pm 394$ & $2531 \pm 551$ & $1870 \pm 260$ & $2281 \pm 278^{*}$ \\
\hline
\end{tabular}

A análise de regressão linear apresentada na Tabela 3 inclui quatro diferentes modelos referentes aos dois métodos utilizados (questionário de freqüência e médias dos recordatórios de 24 horas) e à análise em separado de consumo de energia e gordura. Nas quatro análises multivariadas, associaram-se positivamente ao IMC a idade e pertencer ao grupo de funcionários de nível médio e de apoio comparados aos professores. Ou seja, independentemente do consumo de gordura, ou energia e do dispêndio energético, ocupação e idade estiveram associadas ao IMC. Ainda, nesta análise, um aumento de 500 calorias no gasto energético diário estimado eqüivaleria a uma redução de, aproximadamente, $2 \mathrm{~kg}$ no peso de um indivíduo com $1,60 \mathrm{~m}$.
Para os dois métodos usados para avaliação de consumo, a ingestão de gordura associou-se, significativamente com o IMC $(\mathrm{p}<0.05)$, porém esta associação foi modificada pelo sexo. $\mathrm{O}$ consumo calórico associou-se com o IMC somente quando medido pelo QFCA.

Uma vez que houve interação entre o consumo de gordura e de energia com o sexo (termo energia*sexo) a estimativa do IMC em função do consumo só pode ser interpretada segundo sexo, sendo apresentada na Figura 1 para o consumo medido pelo QFCA. Tanto para o consumo de gordura, quanto para o consumo de energia observa-se redução do IMC com o aumento de consumo para as mulheres e ligeiro aumento do IMC com o aumento de consumo para os homens. 
Tabela 3. Coeficientes ( $\beta$ ), erro-padrão (EP) e valor de $\mathrm{p}(\mathrm{p})$ associados à regressão linear multivariada, utilizando dois métodos de coleta de dados.

\begin{tabular}{|c|c|c|c|c|c|c|}
\hline \multirow[b]{2}{*}{ Variáveis } & \multicolumn{3}{|c|}{ QFCA } & \multicolumn{3}{|c|}{ Recordatórios 24 h } \\
\hline & $\beta$ & EP & $\mathrm{p}$ & $\beta$ & EP & $\mathrm{p}$ \\
\hline & \multicolumn{6}{|c|}{ Consumo de energia } \\
\hline Idade (anos) & 0,070 & 0,035 & 0,05 & 0,090 & 0,033 & 0,009 \\
\hline Grupo (Superior/médio e apoio) & $-2,52$ & 0,79 & 0,002 & $-2,33$ & 0,86 & 0,008 \\
\hline Gasto energético/peso & $-2,08$ & 0,58 & 0,0005 & $-2,19$ & 0,60 & 0,0005 \\
\hline Sexo (feminino/masculino) & 4,51 & 2,03 & 0,02 & 3,75 & 2,65 & 0,16 \\
\hline Energia (100 kcal) & 0,28 & 0,14 & 0,04 & 0,23 & 0,18 & 0,20 \\
\hline \multirow[t]{2}{*}{ Energia * sexo } & $-0,23$ & 0,10 & 0,02 & $-0,20$ & 0,14 & 0,17 \\
\hline & \multicolumn{6}{|c|}{ Consumo de gordura } \\
\hline Idade (anos) & 0,075 & 0,033 & 0,02 & 0,085 & 0,033 & 0,01 \\
\hline Grupo (superior/médio e apoio) & $-2,52$ & 0,79 & 0,004 & $-2,03$ & 0,88 & 0,02 \\
\hline Gasto energético/peso & $-2,30$ & 0,57 & 0,0008 & $-2,26$ & 0,56 & 0,0003 \\
\hline Sexo (feminino/masculino) & 3,04 & 2,03 & 0,04 & 3,86 & 2,02 & 0,06 \\
\hline Gordura (10g) & 0,74 & 0,36 & 0,04 & 0,70 & 0,40 & 0,08 \\
\hline Gordura * sexo & $-0,66$ & 0,28 & 0,02 & $-0,60$ & 0,31 & 0,05 \\
\hline
\end{tabular}

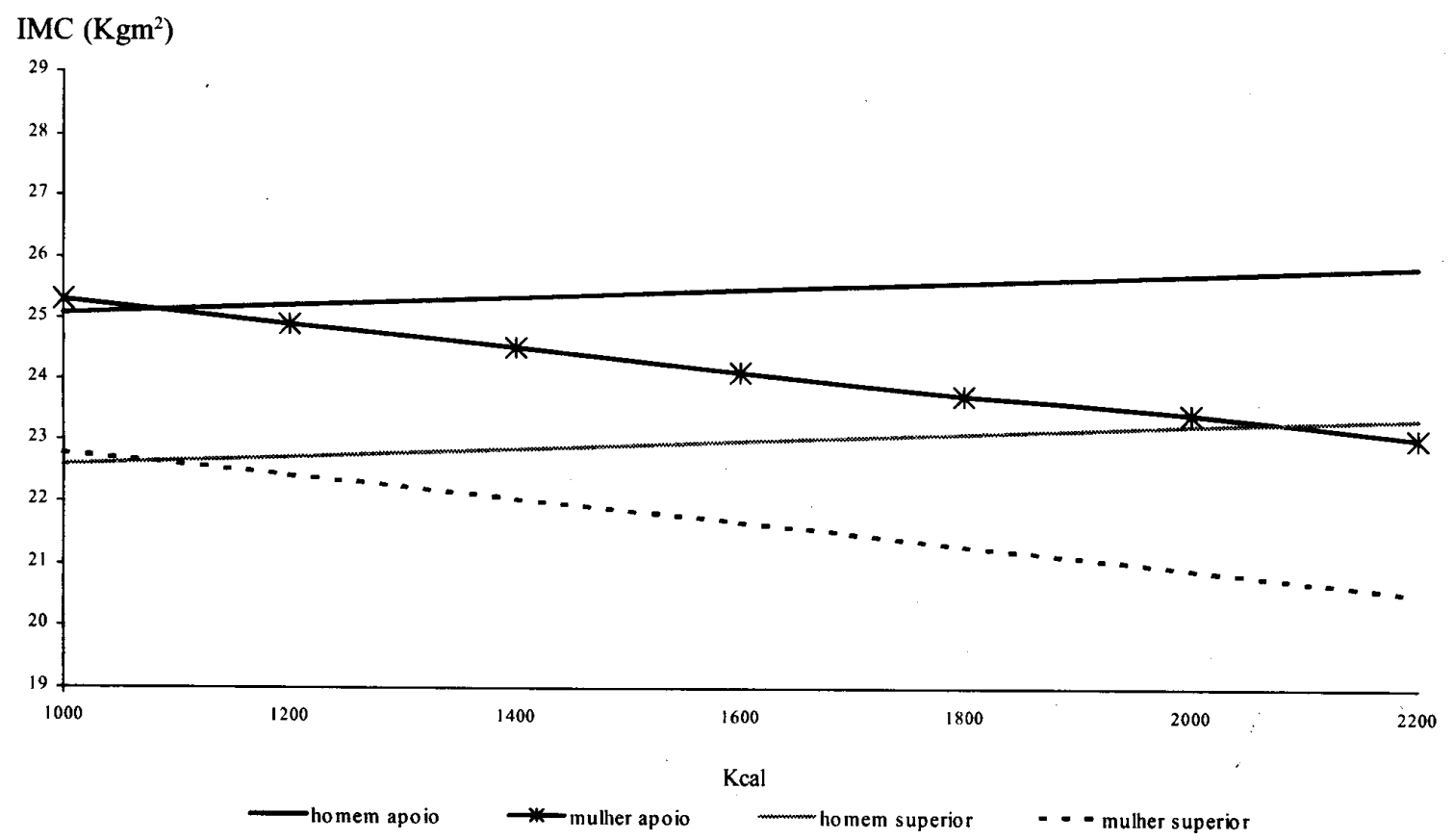

Figura 1A. Consumo de energia. (valores estimados a partir da análise de regressão linear multivariada apresentada na Tabela 3). 


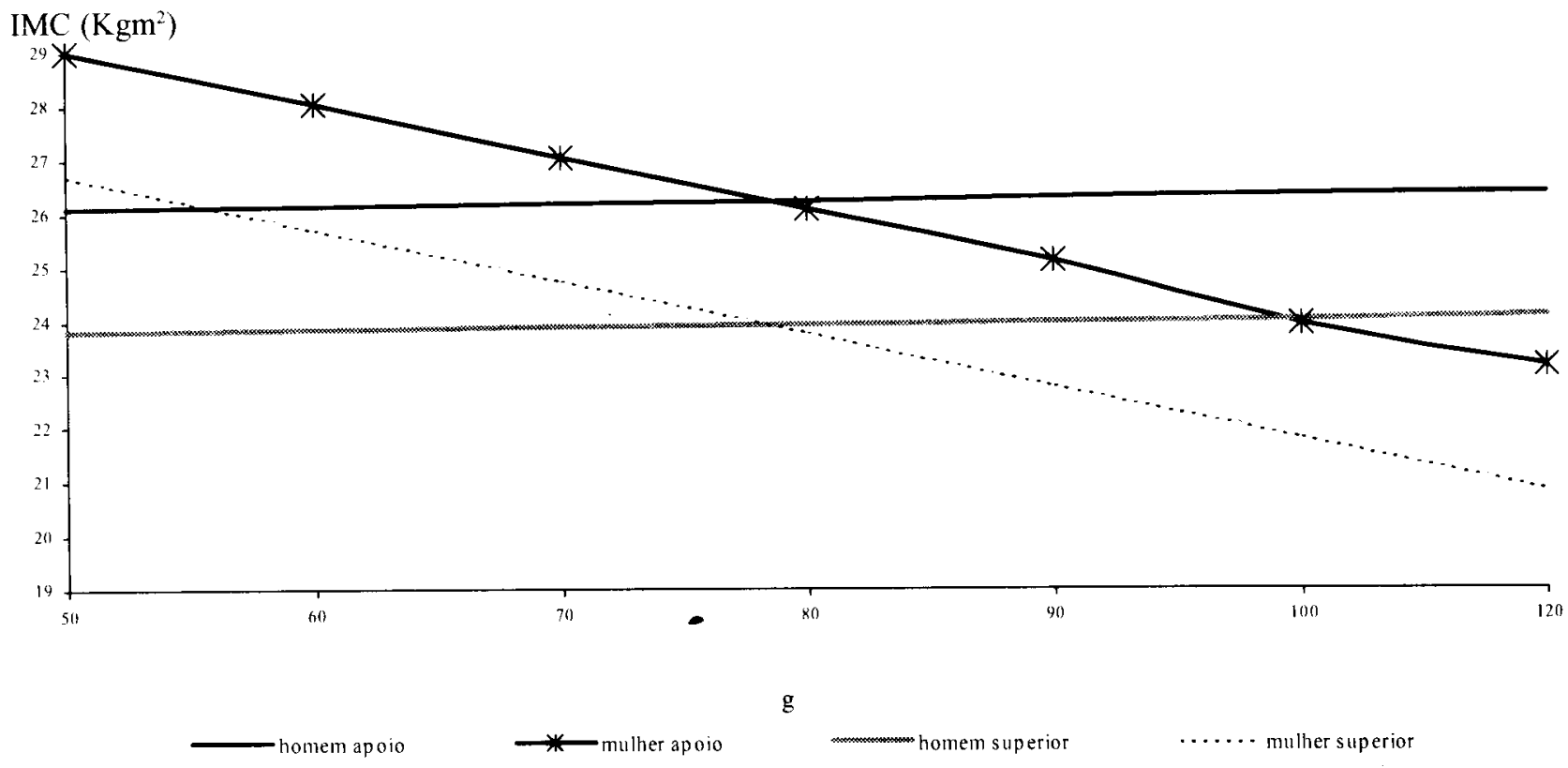

Figura 1B. Consumo de gordura (valores estimados a partir da análise de regressão linear multivariada apresentada na Tabela 3).

\section{DISC USSÃO}

A Pesquisa Nacional sobre Saúde e Nutrição (PNSN), realizada em 1989, mostrou que o sobrepeso é um problema importante no Brasil (COITINHO et al., 1991). Os dados deste trabalho, embora referentes a um reduzido número de indivíduos, mostram que o excesso de peso ocorreu com maior freqüência entre trabalhadores de nível médio e de apoio do que entre os de nível superior. Entre os homens do grupo de nível médio e de apoio tanto a prevalência de sobrepeso quanto o valor da prega triciptal foram significativamente maiores. Esta tendência de maior sobrepeso e obesidade entre o grupo de baixo nível socioeconômico, embora em direção contrária à da PNSN, tem sido observada por outros autores nacionais (ANJOS et al., 1992; VEIGA \& SIGULEM, 1994) e aceita de forma incontestável nos países desenvolvidos (SICHIERI et al., 1992). Mesmo a associação encontrada na PNSN já indicava uma redução da prevalência de obesidade entre mulheres nos dois maiores quintis de renda (SICHIERI et al., 1994).

Os resultados obtidos relativos à prática de atividade física são interessantes no sentido de indicar uma diferença entre os grupos em relação a caminhar para o trabalho, com média diária próxima dos 30 minutos no grupo denível médio e de apoio e próxima a 10 minutos entre o grupo de nível superior. Atividade física associada ao lazer foi mais freqüente entre as mulheres de nível superior. As análises multivariadas indicaram que o gasto energético estimado, que leva em conta a freqüência e duração das atividades e que mede grosseiramente o real dispêndio de energia, parece ser um dos importantes preditores do IMC. Observa-se que na análise multivariada o gasto energético foi avaliado por unidade de peso indicando que atividade física mais intensa associou-se a menor IMC. A associação entre atividade física, consumo alimentar e sobrepeso tem sido discutida por vários autores e JONHSON et al. (1956) mostraram que o baixo consumo de calorias associava-se com obesidade e com atividade física reduzida. WILLETT (1990) discute que embora o gasto associado à atividade física seja pequeno, ele é o maior diferenciador do gasto energético total, dado que as outras frações do gasto energético são mais constantes. WILMORE (1996) considera que a atividade física é mais importante na prevenção do sobrepeso e da obesidade do que no seu tratamento, porque quando a obesidade já se instalou, mudanças na ingestão estão mais associadas à perda de peso que o aumento de atividade física. 
Se entendermos que a atividade física é um importante determinante da obesidade e que ela também determina o consumo alimentar, a associação negativa que encontramos entre consumo de calorias e gorduras e o IMC poderia estar relacionada à atividade física. Contudo, na análise multivariada, ajustada para o gasto energético, a associação negativa persiste entre as mulheres, o que deve-se, provavelmente, a viés de informação entre mulheres com maior IMC. Este viés de informação tem sido claramente mostrado por diferentes autores (PRENTICE et al., 1986; SCHOELLER, 1990; HEITMANN \& LISSNER, 1996). Por outro lado, hipóteses têm sido formuladas, de que alguns grupos populacionais, particularmente aqueles submetidos durante fases de desenvolvimento a alguma restrição, seriam mais propensos a apresentarem maior eficiência metabólica, que se traduziria em acúmulo de energia mesmo com baixo aporte energético.

SALTZMAN \& ROBERTS (1995) em recente revisão sobre o papel do gasto energético na regulação do ganho de peso, concluem que em indivíduos geneticamente suscetíveis ao excesso de peso parece ocorrer menor dispêndio energético associado ao metabolismo de repouso e à atividade física. Estes mecanismos poderiam explicar os nossos dados de baixo consumo entre os trabalhadores de nível socioeconômico menos favorecido e a maior prevalência de sobrepeso encontrada neste grupo.

Outra hipótese que pode ser levantada, tentando explicar esta constatação é o fato de que as mulheres deste estudo sendo mais obesas podem controlar mais sua alimentação na tentativa de reduzir o peso ou de controlar possíveis doenças associadas ao sobrepeso. Pelo fato deste estudo ser de corte transversal, ele não permitiu avaliar claramente as associações com o IMC, dado que a temporalidade entre fatores em estudo e o IMC fica comprometida.

É de grande interesse o achado de que não houve diferença entre os grupos ocupacionais nesta associação, uma vez que mesmo ajustando para ocupação a associação negativa entre consumo e IMC persiste. Poder-se-ia esperar que entre as mulheres de nível superior ocorresse maior subestimação de consumo, fruto de uma imposição de padrões de magreza de maior aceitação social. A tendência observada na Figura 1 sugere, inclusive, uma maior redução do IMC com o aumento de consumo de gordura entre as mulheres do grupo de apoio do que entre as mulheres do nível superior.

Embora este estudo não tenha sido desenhado com a finalidade de testar a associação entre consumo alimentar, atividade física e IMC, os resultados apresentados sugerem que o gasto energético estimado é um importante preditor do IMC. Adicionalmente, e em consonância com a literatura, encontramos que os grupos de menor nível socioeconômico, o gênero feminino e os de maior faixa etária apresentam maior risco de sobrepeso. As associações encontradas para o consumo alimentar parecem estar influenciadas por importante viés de informação entre as mulheres.

Os dados aqui apresentados podem ser reflexo das mudanças experimentadas no país nos últimos anos; a crescente urbanização, a expansão do setor terciário na economia e as mudanças na produção agrícola e industrial de alimentos, favoreceram não somente alterações nos hábitos de alimentação mas também no padrão de atividade física, concorrendo para a sedentarização de expressiva parcela da população e aumentando a oferta e reduzindo o preço de alimentos ricos em energia elipídeos, como relatam MONDINI \& MONTEIRO (1994).

Este estudo sugere que nível socioeconômico e o gasto energético são preditivos de obesidade e que, possivelmente as mulheres obesas subestimam seu consumo alimentar. Outras investigações devem ser realizadas, de preferência de caráter longitudinal, para que as relações entre consumo alimentar, atividade física e obesidade sejam melhor exploradas e considerando ainda variáveis marcadoras de nível socioeconômico para que possamos aprofundar as hipóteses levantadas por este estudo.

\section{REFERÊNCIAS BIBLIOGRÁFICAS}

ANJOS, L.A., SILVA, D.O., SERRÃO, S.A. Vigilância nutricional em adultos: experiência de uma unidade atendendo população favelada. Cadernos de Saúde Pública, Rio de Janeiro, v.8, n.1, p.50-56, 1992.

BRAITMAN, L.E., ADLIN, E.V., STANTON, J.L. Obesity and caloric intake: the National Health and Nutrition Examination Survey of 1971-1975(HANES I). JChronic Dis. v.38, n.9, p.727-32, 1985.

COITINHO, D.C., LEÃO, M.M., RECINE, E., SICHIERI, R. Condições nutricionais da população brasileira. Brasília : INAN, 1991. 39p. (Pesquisa Nacional sobre Saúde e Nutrição). 
FEHILY, A.M., YARNELL, W.G., ELWOOD P.C. Diet and incident heart disease: the Caerphilly study. British Journal of Nutrition, London, v.69, n.2, p.303-314,1993.

GORDON, T., KAGAN, A., GARCIA-PALMIERI, M., KANNEL, W.B., ZUKEL, W.J., TILLOTSON, J., SORLIE, P., HJORTLAND, M. Diet and its relation to coronary heart disease and death in three populations. Circulation, Dallas, v.63, n.3, p.500-515, 1981.

HEITMANN, B.L., LISSNER, L. Dietary underreporting by obese individuals: is it specific or non-specific? British Medical Journal, London, v.311, n.7011, p.986989, 1996.

JOHNSON, M.L., BURKE, B.S., MAYER, J. Relative importance of inactivity and overeating in the energy balance of obese high school girls. American Journal ofClinical Nutrition, Bethesda, v.4, n.1, p.37-44, 1956.

LICHTMAN, S.W., PISARSKA, K., BERMAN, N. Discrepancy between self-reported and actual caloric intake and exercise in obese subjects. New England Journal of Medicine, Boston, v.327, n.27, p.18931898, 1992

MARTIN-MORENO, C., BOYLE, P., GORGOJO, L., MAISONNEEUVE, P., FERNANDEZ-RODRIGUEZ, J.C. Development and validation of a food frequency questionnaire in Spain. International Journal of Epidemiology, London, v.22, n.3, p.512-519, 1993.

MONDINI, L., MONTEIRO, C.A. Mudanças no padrão de alimentação da população urbana brasileira (1962-1988). Revista de Saúde Pública, São Paulo, v.28, n.6, p.433-439, 1994.

MONTEIRO, C.A., IUNES, R.F., TORRES, A.M. A evolução do país e de suas doenças: síntese, hipóteses e implicações. In: MONTEIRO C.A. (Org.) Velhos e novos males da saúde no Brasil: a evolução do país e de suas doenças. São Paulo : Hucitec, 1995. 372p.

NATIONAL RESEARCH COUNCIL (USA). Recommended dietary allowances 10.ed. Washington DC : National Academy of Sciences, 1989a. 284p.

NATIONAL RESEARCH COUNCIL (USA). Obesity and eating disorders. In: NATIONAL RESEARCH COUNCIL (USA). Diet and health: implications for reducing chronic disease risk, Washington DC, 1989b. p.563-592.

PINHEIRO, A.B.V., LACERDA, E.M.A., BENZECRY, E.H., GOMES, M.C.S., COSTA, V.M. Tabela de avaliação de consumo alimentar em medidas caseiras. Rio de Janeiro : UFRJ. 1993. 74p. (Departamento de Nutrição).
PRENTICE, A.M., BLACK, A.E., COWARD, W.A., DAVIES, H.L., GOLDBERG, G.R., ASFORD, J., SAWYER, M., WHITEHEAD, R.G. High levels of energy expenditure in obese women. British Medical Journal, London, v.292, n.6526, p.983-987, 1986.

PYRRO, A.S., LACERDA, E.M.A. SISNUT - sistema de nutrição: manual do usuário. Rio de Janeiro : UFRJ, 1994.15p.

ROMIEU, I., WILLETT, W.C., STAMPFER, G.A., COLDITZ, L., SAMPSON, B., ROSNER, C.H., HENNEKENS, C.H., SPEIZER, F.E. Energy intake and other determinants of relative weight. American Journal of Clinical Nutrition, Bethesda, v.47, n.3, p.406-412, 1988.

SALTZMAN, E., ROBERTS, S.V. The role of energy expenditure in energy regulation. Nutrition Reviews, New York, v.53, n.8, p.209-220, 1995.

SCHOELLER, D.A. How accurate is self-reported dietary energy intake? Nutrition Reviews, New York, v.48, n.10,p.373-379, 1990.

SICHIERI, R., EVERHART, J., HUBBARD, U.S. Relative weight classification in the assessment of underweight and overweight in the United States. International Journal of Obesity and Related Metabolic Disorders, Hampshire, v.16, p.303-312, 1992.

SICHIERI, R., COITINHO, D.C., LEÃO, M.M., RECINE, E., EVERHART, J. High temporal, geographic and income variation in body mass index among adults in Brasil. American Journal of Public Health, Washington DC, v.84,n.5, p.793-798, 1994.

SICHIERI, R., EVERHART, J. Gender difference in the association of reported energy consumption with energy requeriment in a sample of Brazilian workers. American Journal of Clinical Nutrition, Bethesda, v.61, n.4, p.904, 1995a.

SICHIERI, R. et al. Validação de um questionário de consumo alimentar. In: CONGRESSOBRASILEIRO, 3., CONGRESSO IBERO-AMERICANO, 2., CONGRESSO LATINO-AMERICANO DE EPIDEMIOLOGIA, 1., Salvador, de24a28deabrilde1995, Salvador. Anais..., Salvador, 1995b. 457p.

STATISTICAL ANALYSIS SISTEM-SAS. Version 6.4. [s.l.] : SAS Intitute Inc. Cary. 1995.956p.

VEIGA, G.V., SIGULEM, D.M. Avaliação da composição corporal através de medidas antropométricas do estado nutricional de adolescentes em dois niveis sócio-econômicos. Jornal de Pediatria, Rio de Janeiro, v.70, n.4, p.206-214, 1994. 
WORLD HEALTH ORGANIZATION. Energy and protein requirements. Geneva, 1985. 452p. (Technical Report Series, 724).

WILLETT, W.C. Nutritional Epidemiology. Oxford : Oxford University Press, 1990. 396p. (Monographs in Epidemiology and Bioestatistic, v.15)

WILLETT, W.C. Future directions in the development of food-frequency questionnaires. American Journal of Clinical Nutrition, Bethesda, v.59, n.1, p.171S-174S, 1994.
WILMORE, J.H. Increasing physical activity: alterations in body mass and composition. American Journal of Clinical Nutrition, Bethesda, v.63, n.35, p.456S-460S, 1996.

WINKLEBY, M.A., JATULIS, D.E., FRANK, E., FORTMANN, S.P. Socioeconomic status and health: how education, income, and occupation contribute to risk factors for cardiovascular disease. American Journal of Public Health, Washington DC, v.82, n.6, p.816-820,1992.

Recebido para publicação em 28 de maio de 1997 e aceito em 2 de abril de 1998. 